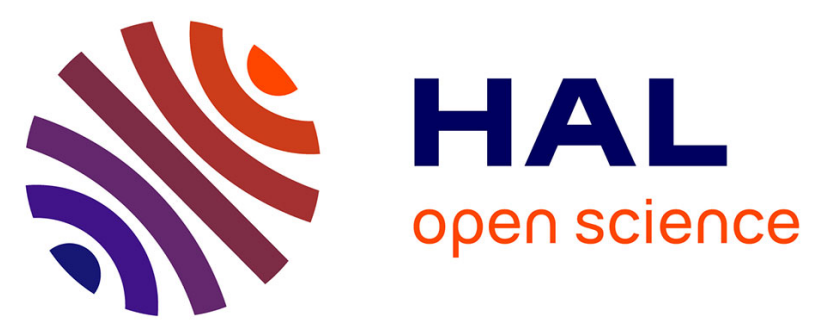

\title{
On the feasibility of making intelligent antenna selection decision in IEEE 802.15.4 wireless sensor networks
}

Mubashir Husain Rehmani, Abderrezak Rachedi, Stéphane Lohier, Thierry Alves, Benoit Poussot

\section{- To cite this version:}

Mubashir Husain Rehmani, Abderrezak Rachedi, Stéphane Lohier, Thierry Alves, Benoit Poussot. On the feasibility of making intelligent antenna selection decision in IEEE 802.15.4 wireless sensor networks. ComComAP'2013, Apr 2013, Hong Kong, China. pp.41 - 46, 10.1109/ComComAp.2013.6533606 . hal-00788125

\section{HAL Id: hal-00788125 \\ https://hal.science/hal-00788125}

Submitted on 23 Mar 2014

HAL is a multi-disciplinary open access archive for the deposit and dissemination of scientific research documents, whether they are published or not. The documents may come from teaching and research institutions in France or abroad, or from public or private research centers.
L'archive ouverte pluridisciplinaire HAL, est destinée au dépôt et à la diffusion de documents scientifiques de niveau recherche, publiés ou non, émanant des établissements d'enseignement et de recherche français ou étrangers, des laboratoires publics ou privés. 


\title{
On the Feasibility of making Intelligent Antenna Selection Decision in IEEE 802.15.4 Wireless Sensor Networks
}

\author{
Mubashir Husain Rehmani ${ }^{\star \ddagger}$, Abderrezak Rachedi ${ }^{\star}$, Stéphane Lohier ${ }^{\star}$ \\ ${ }^{\star}$ Université Paris-Est Marne-la-Vallée, LIGM, PasNet, France \\ $\ddagger$ COMSATS Institute of Information Technology, Pakistan \\ rehmani@univ-mlv.fr, rachedi@univ-mlv.fr, lohier@univ-mlv.fr \\ ${ }^{\dagger}$ Thierry Alves, ${ }^{\dagger}$ Benoit Poussot \\ †Université Paris-Est Marne-la-Vallée, ESYCOM, France \\ talves@univ-mlv.fr, benoit.poussot@univ-mlv.fr
}

\begin{abstract}
In this paper, we study the feasibility of making intelligent antenna selection decision in IEEE 802.15.4 wireless sensor networks. This study provides us the basis to design and implement software defined intelligent antenna switching capability to wireless sensor nodes based on link quality metric, such as Received Signal Strength Indicator (RSSI). As a first step, we discuss in detail the results of our newly designed radio module (Inverted-F Antenna) for $2.4 \mathrm{GHz}$ bandwidth wireless sensor networks. In the second step, we propose an intelligent antenna selection strategy to exploit antenna diversity. To evaluate, we consider TelosB motes and compare the performance of the built-in TelosB antenna with our proposed external antenna in both laboratory and realistic environments. Experimental results confirm the effectiveness of the proposed radio module over the built-in radio module of the TelosB motes.
\end{abstract}

Keywords-Wireless sensor networks, IEEE 802.15.4, ZigBee, RSSI, Antenna

\section{INTRODUCTION}

Wireless Sensor Networks (WSNs) can be seen everywhere and are now becoming the part of our daily life. The application of these WSNs ranges from environmental monitoring like wildlife tracking, habitat monitoring, forest fire detection, mine safety monitoring to military applications like target detection, and tracking [1]. However, the performance of these WSNs depends upon the quality of the wireless link, the built-in antenna available on the sensor device, and the antenna diversity. In WSNs, there are several application scenarios where a clear line-of-sight (LOS) between the sender and receiver is not present [2], [3]. This results in fading of the signal and causes multipath propagation. Furthermore, the signal between transmitterreceiver pair is prone to attenuation and distortion, resulting in packet losses. The main reason of attenuation and

\footnotetext{
This work is part of the project entitled "Wireless Sensor Networks for vehicle and pedestrian traffic assessment applications", funded by the University of Paris Est under the PPS program.
}

distortion is due to path loss, multipath fading, radiation characteristics, antenna orientation, and Doppler effects and are highly dependent upon the location and surrounding of the transmitter-receiver pair [4]. Antenna diversity is a way in which two antennas are attached with the sensor node to improve the quality and reliability of the wireless link [3].

In IEEE 802.15.4, the quality of the link is measured by two metrics: Link Quality Indicator (LQI) and Received Signal Strength Indicator (RSSI). These two metrics are offered by the IEEE 802.15.4 physical layer, which can then be used by the routing layer to select good quality routes. For instance, the impact of LQI-Based routing metrics on the performance of a One-to-One routing protocol for IEEE 802.15.4 Multihop Networks has been studied by the authors in [5]. The authors in [6] provided a detailed study on the comparison of LQI and RSSI metrics. However, aforementioned works [5], [6] did not consider the antenna diversity. Recently, the authors in [7] showed that significant gain can be achieved through antenna diversity but their work mainly focus on directional antennas for smart-phone like mobile devices. In [8], the authors focus on target coverage problem using directional sensor and antennas.

Based on link quality metric RSSI, our ultimate goal is to design and implement software defined intelligent antenna switching capability to wireless sensor nodes. More precisely, we want to attach an external antenna with the sensor nodes besides the built-in antenna to achieve antenna diversity. Then, based on wireless link condition, the sensor node switch to the appropriate antenna for communication.

Our Contributions: This paper makes the following contributions:

- As a first step, we design a new radio module, an Inverted-F Antenna for $2.4 \mathrm{GHz}$ bandwidth wireless sensor networks. In fact, we study the feasibility of making intelligent antenna selection decision in IEEE 802.15.4 wireless sensor networks. This study will provide us the basis to design and implement software 


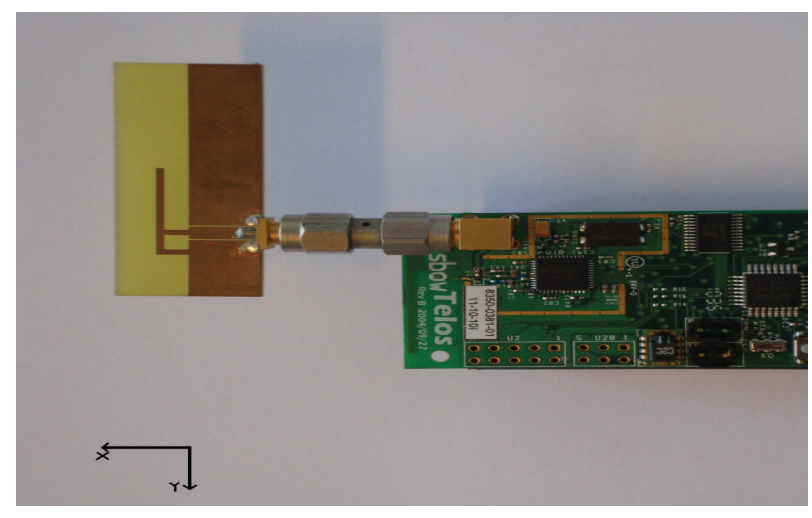

Figure 1. Inverted-F antenna attached with TelosB mote.

defined intelligent antenna switching capability to wireless sensor nodes based on link quality metric (RSSI).

- We perform extensive experiments in both indoor and outdoor environment. We then compare results of our antenna with the built-in TelosB antenna. Experimental results confirm the effectiveness of the proposed radio module over the built-in radio module of the TelosB motes in laboratory and realistic environments.

- In the second step, we propose an intelligent antenna selection strategy to exploit antenna diversity. In this strategy, wireless sensor nodes predict the values of RSSI of built-in and external antenna and make the antenna selection decision adaptively. In fact, the proposed antenna selection strategy is based on Exponentially Weighted Moving Average (EWMA) and rely on the historical observations of the RSSI values of built-in and external antenna.

\section{INVERTED-F ANTENNA (IFA) DESIGN}

According to the TelosB datasheet [11], we have implemented an SubMiniature version A (SMA) connector in order to connect our small external antenna. This antenna is called as Inverted-F Antenna (IFA). Fig. 1 shows Inverted-F antenna attached with TelosB mote. The details regarding the design and connection procedure of our antenna with the TelosB mote can be found in [10].

In the next section, we analyze the performance of IFA antenna and the built-in antenna and study the behaviour of RSSI in different environments.

\section{Performance Analysis of Built-In And EXTERNAL ANTENNA}

We first describe the methodology in this section and then proceed with the performance analysis.

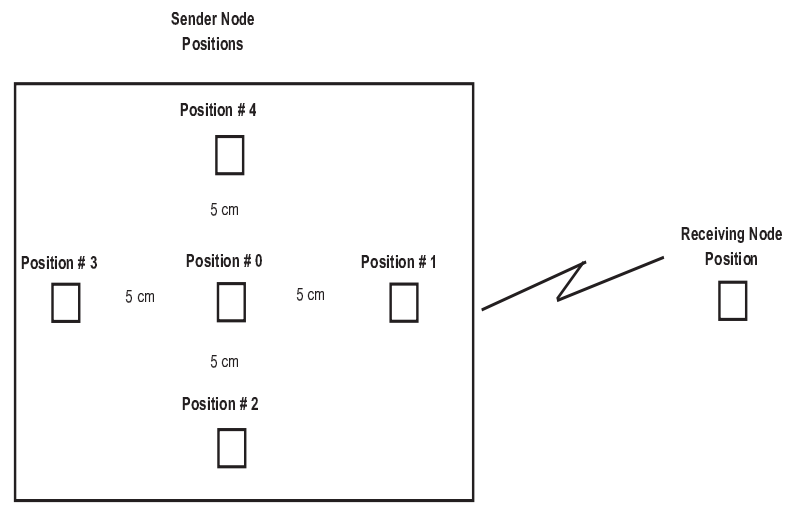

Figure 2. Sender and Receiving nodes positions.

Methodology: Since our goal is to consider RSSI in making the antenna selection decision, we first perform extensive experiments to study the behaviour of RSSI in four different environments, namely, (1) Indoor Office Environment, (2) Outdoor Garden Environment, (3) Reverberation chamber, and (4) Anechoic chamber. Note that the reverberation chamber is used to simulate indoor environment with multipath and the anechoic chamber is used to simulate outdoor environment.

Implementation Setup: We consider Telosb motes [12], which are equipped with Chipcon CC2420 radio module [13]. The radio module CC2420 in its new versions is used in many radio devices. The transceiver of TelosB motes operate in $2.4 \mathrm{GHz}$ band. We use RSSI as a link quality metric to study the performance of our newly designed antenna. The RSSI provides the signal strength at the receiver (in $\mathrm{dBm})[6]$.

We consider two types of TelosB motes in our experiment: (1) sink node, which is connected with the serial port of the Laptop, and (2) the sending mote, which sends packet with a time delay of $500 \mathrm{~ms}$. Total 100 packets were sent by the sending mote. As soon as the sink node receives packet by the sending mote, it saves the RSSI value of each corresponding packet. The sink node and the sender node is placed 1 feet and 1 meter apart. We carried out our experiment at 3 different power levels: $0 \mathrm{dBm},-10 \mathrm{dBm}$ and $-25 \mathrm{dBm}$. To change the transmission power, we change the default transmission power of CC2420 i.e., $0 \mathrm{dBm}$ (CC2420_DEF_RFPOWER = 31), to $-10 \mathrm{dBm}$ and $-25 \mathrm{dBm}$, by changing the register values to 11 and 3, respectively. In order to study the impact of LOS and multipath on RSSI for both antennas, we place the sender node at five different locations, while the receiving node's position was fixed (cf. Fig. 2). We now describe the results for each environment.

\section{A. Indoor Office Environment}

We first consider indoor office environment in which we have walls, computers, tables, and other reflecting material. Moreover, we also have human activity and the experiments 


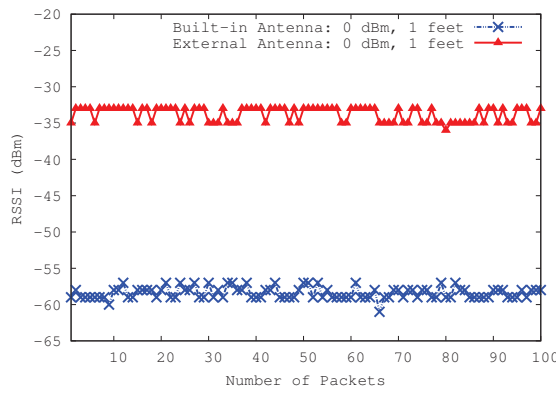

(a)

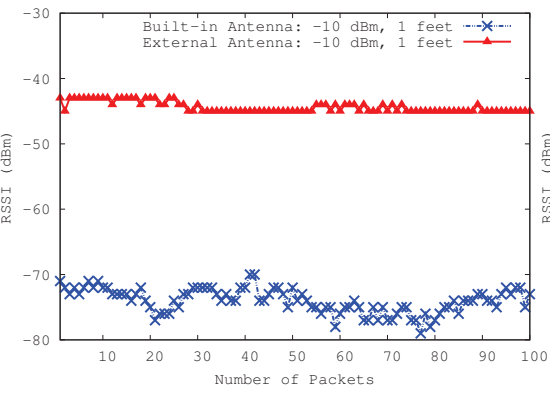

(b)

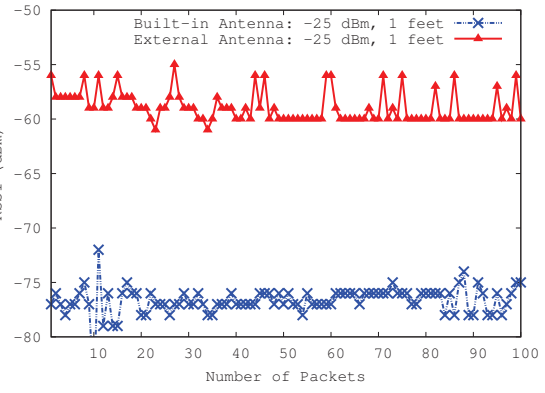

(c)

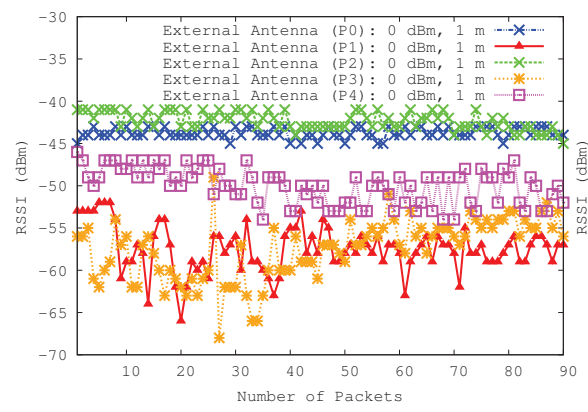

(d)

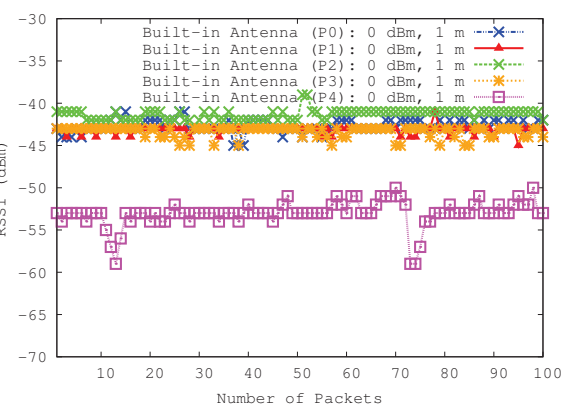

(e)

Figure 3. Number of Packets and RSSI in Indoor office environment. (a) $0 \mathrm{dBm}$ Power. (b) $-10 \mathrm{dBm}$ Power. (c) $-25 \mathrm{dBm}$ Power. (d) External antenna placed at five different locations with $0 \mathrm{dBm}$ Power. (e) Built-in antenna placed at five different locations with 0 dBm Power.

were conducted in the presence of a single person.

Fig. 3 compares the RSSI values of built-in antenna and external IFA antenna for each received packet with $0 \mathrm{dbm}$, $10 \mathrm{dBm}$ and $-25 \mathrm{dBm}$ power levels. Results in Fig. 3(a), 3(b) and 3(c) were taken when the distance between the sender node and the receiving node was 1 feet. While Fig. 3(d) and 3(e) shows the results when the sender node and the receiving node were 1 meter apart. Note that in Fig. 3(d) and 3(e), the sender node is placed at five different locations (cf. Fig. 2).

The IFA antenna provides higher power levels i.e., RSSI values at the receiver. More precisely, when power level is $0 \mathrm{dBm}$, the external IFA antenna provide $-33.81 \mathrm{dBm}$ power compared to $-58.4 \mathrm{dBm}$ power for the built-in antenna, i.e., the gain of $25 \mathrm{~dB}$. When power level is $-10 \mathrm{dBm}$, the external IFA antenna provide $-44.41 \mathrm{dBm}$ power compared to -74.0 $\mathrm{dBm}$ power for the built-in antenna, i.e., the gain of $30 \mathrm{~dB}$. And when the power level is $-25 \mathrm{dBm}$, the external IFA antenna provide $-58.95 \mathrm{dBm}$ power compared to $-76.67 \mathrm{dBm}$ power for the built-in antenna, i.e., the gain of around 17.75 $\mathrm{dB}$. As mentioned earlier, to study the affect of LOS and multipath, we consider five different positions for the sender node. In addition, we also increase the distance between the sender node and receiving node to 1 meter. We now observe the RSSI values for the external and built-in antenna in Fig. 3(d) and 3(e). As can be seen in the figure, the RSSI value is different for every position (cf. Fig. 2). This is due to the multipath affect and due to the absence of clear line of sight between the sender and the receiver nodes. In summary, results in Fig. 3(a), 3(b) and 3(c) confirm that the newly designed IFA antenna can provide good link reliability, suitable for wireless radio communication in IEEE 802.15.4 based WSNs.

We also conclude that when the sender and receiver nodes are at short distance, we observe the different RSSI values for the two antennas (cf. Fig. 3(a), 3(b) and 3(c)). On the other hand, when the sender and receiver nodes are at long distance, the RSSI values received at both the antennas fluctuates (cf. Fig. 3(d) and 3(e)). This is because of multipath fading and scattering effects, which ultimately changes the polarization of waves.

\section{B. Reverberation Chamber}

Reverberation chambers are used to study the effects of multi-path propagation environments [14].

We compare the RSSI values of built-in antenna and external IFA antenna in Reverberation Chamber, for each received packet with $-25 \mathrm{dBm}$ power level, as shown in Fig. 4. More precisely, results in Fig. 4 were taken when the nodes were 1 meter apart and the sender node is placed at two different locations (cf. Fig. 2).

It can be clearly seen in Fig. 4 that RSSI values in Reverberation chamber highly fluctuates. This is due to the highly dynamic environment (Azimuth Stirrer rotation at 2 rpm). This results in multi-path and thus we have varying RSSI values for both antennas. Another important point that need to be noted over here is that the RSSI value for both antennas is time varying, means, for any time instant $t$, 


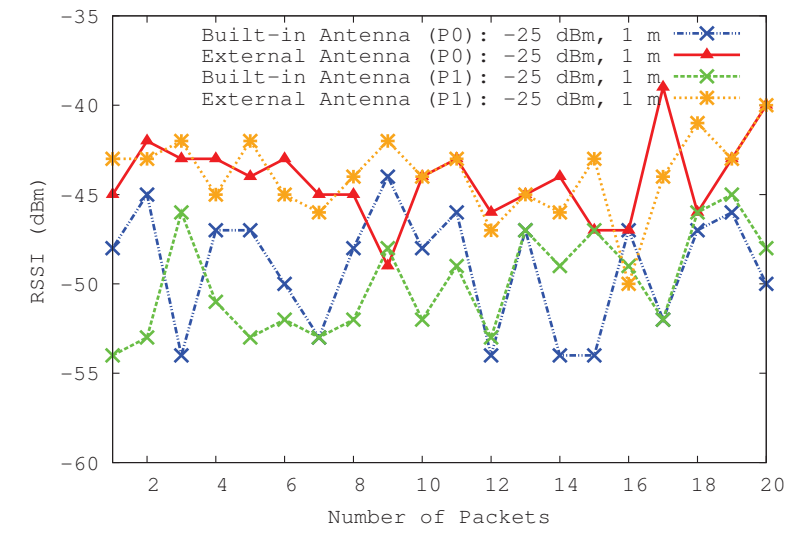

Figure 4. Number of Packets and RSSI in Reverberation chamber environment.

external antenna is performing good, while for time instant $t+1$, built-in antenna gives good performance. Thus, we plan to use this spatio-temporal variation in RSSI to select appropriate antenna (cf. Section IV for more details).

\section{RF Anechoic Chamber}

In Fig. 5, we compare the RSSI values of built-in antenna and external IFA antenna in Anechoic Chamber, for each received packet with $0 \mathrm{dbm},-10 \mathrm{dBm}$ and $-25 \mathrm{dBm}$ power levels. Results in Fig. 5(a), 5(b) and 5(c) were taken when the nodes were placed 1 meter apart, while Fig. 5(d) and 5(e) shows the results when the nodes were 1 meter apart and the sender node is placed at five different locations (cf. Fig. 2).

Figure 5 witness that the external antenna outperforms the built-in antenna of TelosB mote. More precisely, with $0 \mathrm{dBm}$ power (cf. Fig. 5(a)), the RSSI of external antenna is -45.51 $\mathrm{dBm}$ and the RSSI of built-in antenna is $-53.94 \mathrm{dBm}$ i.e., the gain of $8.5 \mathrm{~dB}$. Similarly, with $-10 \mathrm{dBm}$ power (Fig. 5(b)), the RSSI of external antenna is $-54.96 \mathrm{dBm}$ and the RSSI of built-in antenna is $-64.3 \mathrm{dBm}$ i.e., the gain of $9.3 \mathrm{~dB}$. The same behaviour can be observed in Fig. 5(c), when the power is $-25 \mathrm{dBm}$.

\section{Outdoor Garden Environment}

In outdoor garden environment, we conduct experiments in the absence of any reflecting material except the ground of the garden.

We compare the RSSI values of built-in antenna and external IFA antenna in Outdoor Garden Environment, for each received packet, with $0 \mathrm{dbm},-10 \mathrm{dBm}$ and $-25 \mathrm{dBm}$ power levels, as can be seen in Fig. 6(a), 6(b) and 6(c). More precisely, results in Fig. 6(a), 6(b) and 6(c) were taken when the nodes were places 1 meter apart. While Fig. 6(d) and 6(e) shows the results when the nodes were 1 meter apart and the sender node is placed at five different locations (cf. Fig. 2).

In the outdoor garden environment, the IFA antenna provides higher power levels i.e., RSSI values at the receiver. More precisely, when power level is $0 \mathrm{dBm}$, the external
IFA antenna provide $-47 \mathrm{dBm}$ power compared to $-62 \mathrm{dBm}$ power for the built-in antenna, i.e., the gain of $15 \mathrm{~dB}$. When power level is $-10 \mathrm{dBm}$, the external IFA antenna provide -57 $\mathrm{dBm}$ power compared to $-73.0 \mathrm{dBm}$ power for the built-in antenna, i.e., the gain of $16 \mathrm{~dB}$. And when the power level is $-25 \mathrm{dBm}$, the external IFA antenna provide $-76 \mathrm{dBm}$ power compared to $-83 \mathrm{dBm}$ power for the built-in antenna, i.e., the gain of around $7 \mathrm{~dB}$.

\section{E. Concluding Remarks}

Results in section III-A, III-B, III-C and III-D shows that when dealing with different environments (office environment, outdoor garden environment, reverberation chamber, and anechoic chamber), we observed fluctuation on RSSI values for both antennas. This provide us the basis and motivate us to exploit antenna diversity. Results also confirm that if external antenna is used, the gain of RSSI is significant.

In this paper, we argued that antenna diversity can be used to achieve gain in RSSI. We also noticed that spatio-temporal fluctuations or interference may lead to the degradation of signal power (RSSI). We showed that this happens very frequently and if a proper strategy is designed to change the intelligent antenna selection decision adaptively, we can achieve higher gain.

In the next section, we discuss our proposed EWMA based adaptive intelligent antenna selection strategy.

\section{EWMA BASEd InTELligent Antenna Selection STRATEGY}

EWMA is a one-step prediction technique [15], which has been widely used in different applications, such as anomaly detection [16], bandwidth prediction etc. In EWMA, higher importance is given to the more recent observations. Thus, EWMA is very suitable for predicting the RSSI value on a particular antenna. In fact, our goal is to detect any small variation in the RSSI value through EWMA prediction technique and then use the appropriate antenna.

In order to achieve this goal, we enable the wireless sensor nodes to keep the record of the RSSI values and this record will be served as an input to predict the RSSI value. Initially, no prediction will be done and each wireless sensor node just collect the historical data of RSSI values on different antennas. Once the nodes have some historical data, the receiving node will start predicting the RSSI values for both antennas. The antenna which provides strong predicted RSSI value at time instant $t$ will then be selected for the next reception period, thus achieving the goal of antenna diversity. Fig. 7 shows the decision making by the sensor nodes to select the appropriate antenna according to the varying RSSI value. As can be seen in Fig. 7, first sensor nodes predict the values of RSSI for both built-in and external antenna. If the RSSI value of external antenna is greater than the built-in antenna, sensor node will switch to the external antenna, else vice versa. Then, sensor nodes 


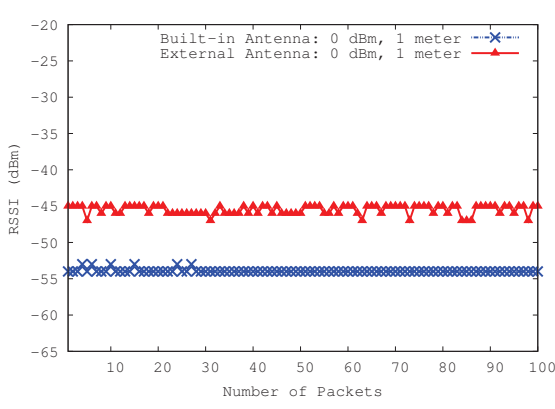

(a)

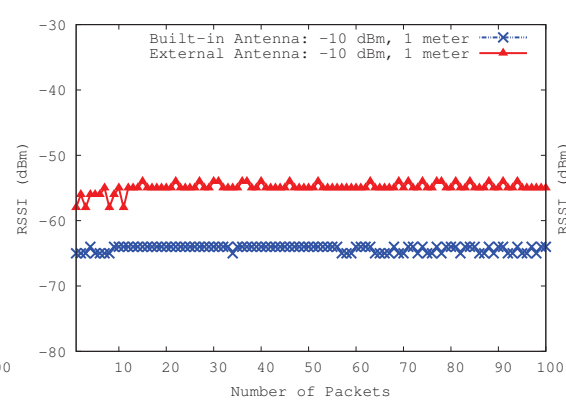

(b)

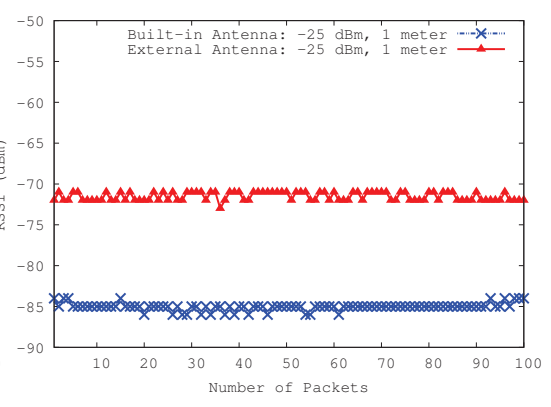

(c)

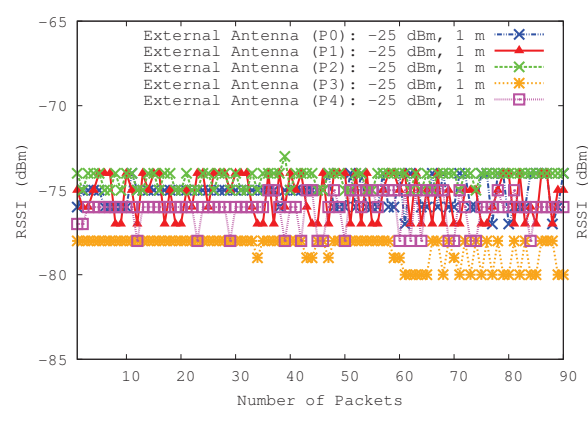

(d)

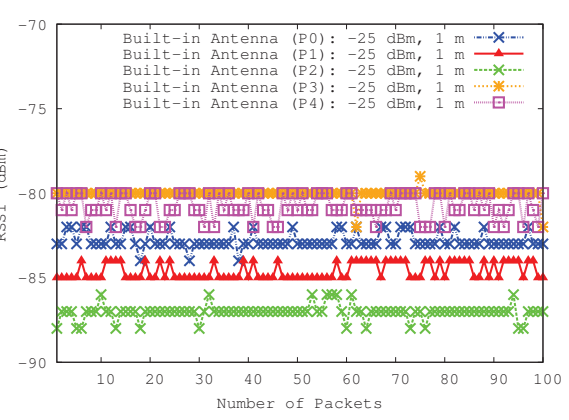

(e)

Figure 5. Number of Packets and RSSI in Anechoic chamber environment. (a) 0 dBm Power. (b) $-10 \mathrm{dBm}$ Power. (c) -25 dBm Power. (d) External antenna placed at five different locations with $-25 \mathrm{dBm}$ Power. (e) Built-in antenna placed at five different locations with $-25 \mathrm{dBm}$ Power.

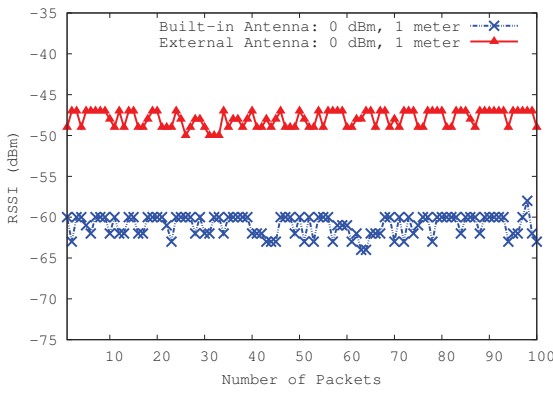

(a)

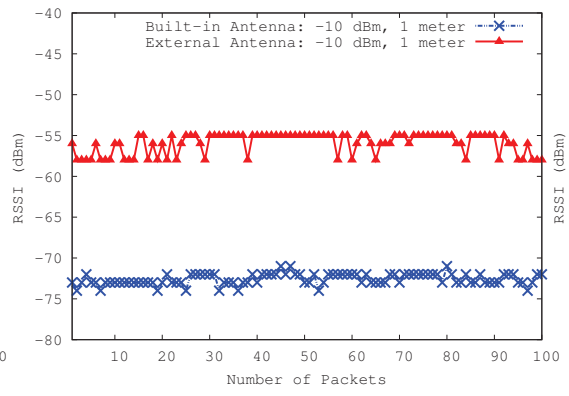

(b)

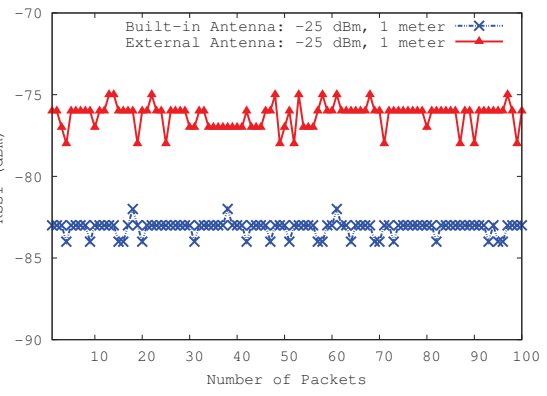

(c)

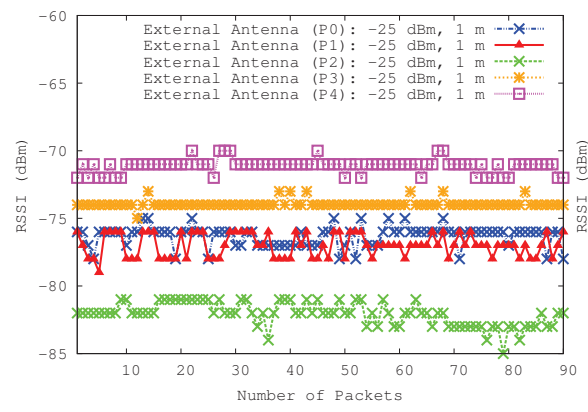

(d)

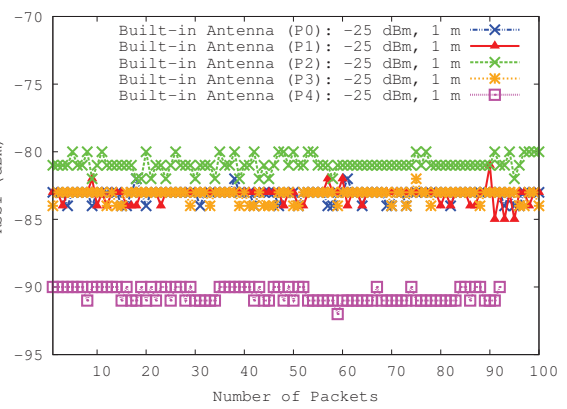

(e)

Figure 6. Number of Packets and RSSI in Outdoor Garden environment. (a) 0 dBm Power. (b) $-10 \mathrm{dBm}$ Power. (c) $-25 \mathrm{dBm}$ Power. (d) External antenna placed at five different locations with $-25 \mathrm{dBm}$ Power. (e) Built-in antenna placed at five different locations with $-25 \mathrm{dBm}$ Power. 
keep these observed values of RSSI in the memory. Finally, these stored RSSI values will be then feedback to predict for the next time.

Let $\triangle R S S I(t)^{E}$ be the mean of historical values of RSSI, $\triangle R S S I$ (t) be the observation at time $\mathrm{t}$ and $\gamma$ is a smoothing factor $(0 \leq \gamma \leq 1)$. The $\operatorname{RSSI}$ value at time $(t+1)$ i.e., $\triangle R S S I(t+1)$ can be calculated using the following formula:

$$
\triangle R S S I(t+1)^{E}=(1-\gamma) \triangle R S S I(t)^{E}+\gamma \triangle R S S I(t)
$$

In this manner, the wireless sensor node can predict the value of RSSI at time instanct $t$ and make an intelligent antenna selection decision. This decision is achieved by implementing an adaptive electronic switch which reacts on the predicted value of RSSI.

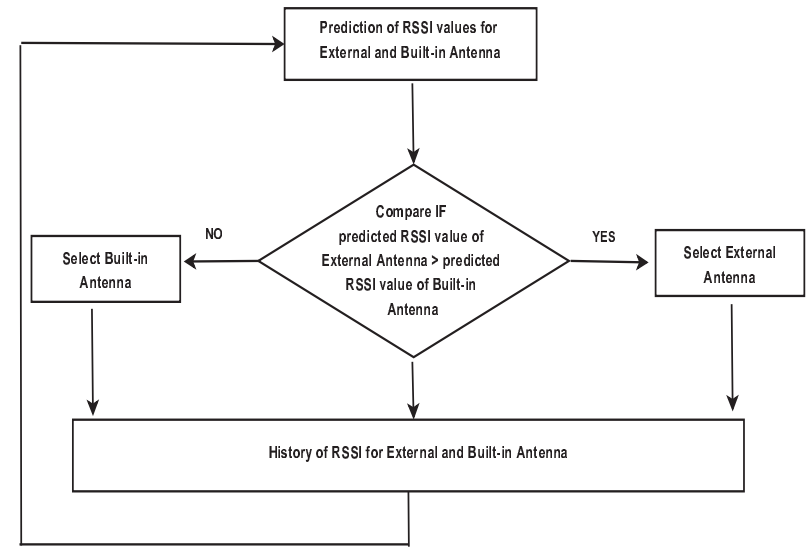

Figure 7. Flow chart shows the decision making by the sensor nodes to select the antenna.

However, it may be possible that due to some abrupt fluctuation in the environment, the RSSI value vary a lot from the mean value of RSSI. This feature is exploited for intrusion detection [9] and for monitoring the human activity. In this paper, we focus on the consistent values of RSSI, instead of identifying the anomaly. In case, any anomaly is detected, the wireless sensor nodes will not take into account those anomalies. In order to identify any anomaly in $\triangle R S S I$ (t), we propose to use two control limits, Upper Control Limit (UCL) and Lower Control Limit (LCL), which can be calculated as:

$$
\begin{gathered}
U C L_{\triangle R S S I}=\triangle R S S I(t)+k s_{\triangle R S S I} \\
L C L_{\triangle R S S I}=\triangle R S S I(t)-k s_{\triangle R S S I} \\
s_{\triangle R S S I}^{2}=\frac{\gamma}{2-\gamma} s^{2}
\end{gathered}
$$

where $s^{2}$ is the variance of the generated and updated data base, which is used to calculate UCL and LCL, and k is set equal to 3 [17].

\section{CONCLUSiOn AND Future Work}

In this paper, we discussed our results of the newly deigned radio module (Inverted F Antenna) for $2.4 \mathrm{GHz}$ wireless sensor networks. We first performed extensive experiments to understand the behaviour of RSSI values for the built-in and external antenna, in both lab and outdoor environments. We then proposed an EWMA based intelligent antenna selection strategy for wireless sensor nodes. Experimental results confirmed the effectiveness of our proposed radio module over the built-in TelosB radio module. In future, we plan to study different routing metrics with antenna diversity under multi-hop network configuration, and efficiency of IFA antenna. Finally, our goal is to integrate software defined switching capability to TelosB motes to change the antenna decision adaptively.

\section{REFERENCES}

[1] Th. Arampatzis, J. Lygeros, S. Manesis, A Survey of Applications of Wireless Sensors and Wireless Sensor Networks, Proceedings of the 2005 IEEE ISIC-MED, pp.719-724, 27-29 June 2005

[2] M. Radenkovic, and B. Wietrzyk, Wireless Mobile Ad-hoc Sensor Networks for Very Large Scale Cattle Monitoring, ASWN'06, Germany, 2006.

[3] Konstantinos Sasloglou, Ian A. Glover, Hock Guan Goh, Kae Hsiang Kwong, Michael P. Gilroy, Christos Tachtatzis, W. Craig Michie, Ivan Andonovic, Antenna and Base-Station Diversity for WSN Livestock Monitoring, Wireless Sensor Network Journal, volume 1, no. 5, pages 383-396, 2009.

[4] T. S. Rappaport, Wireless Communication Principles and Practice, 2nd Edition, Prentice Hall, New Jersey, 1996.

[5] Carles Gomez, Antoni Boix, and Josep Paradells. Impact of lqi-based routing metrics on the performance of a one-to-one routing protocol for IEEE 802.15.4 multihop networks. EURASIP Journal on Wireless Communications and Networking, 6:1-6:20, July 2010.

[6] Kannan Srinivasan and Philip Levis. RSSI is under appreciated. In Proceedings of EmNets, 2006.

[7] Ardalan Amiri Sani, Lin Zhong, and Ashutosh Sabharwal, Directional Antenna Diversity for Mobile Devices: Characterizations and Solutions, MobiCom 2010.

[8] Kai Han, Liu Xiang, Jun Luo, and Yang Liu, Minimum-Energy Connected Coverage in Wireless Sensor Networks with Omni-Directional and Directional Features, MobiHoc 2012.

[9] Pius W.W. Lee, Winston K.G. Seah, Hwee-Pink an, and Zexi Yao, Wireless Sensing Without Sensors - An Experimental Approach, IEEE PIMRC 2009.

[10] Mubashir Husain Rehmani, Thierry Alves, Stephane Lohier, Abderrezak Rachedi, Benoit Poussot, Towards Intelligent Antenna Selection in IEEE 802.15.4 Wireless Sensor Networks, ACM MobiHoc'12, South Carolina, USA, June 11-14, 2012.

[11] Moteiv Corporation, telos datasheet http://www2.ece.ohiostate.edu/ bibyk/ee582/telosMote.pdf

[12] MEMSIC Inc, http://www.memsic.com, 2011.

[13] Chipcon cc2420 datasheet, http://www.chipcon.com, 2011.

[14] Kent Rosengren and Per-Simon Kildal, Study of Distributions of Modes and Plane waves in Reverberation chambers for the characterizaiton fo antennas in a Multi-path environment, Microwave and Optical Technology Letters, Vol. 30, No. 6, September 20, 2001.

[15] S. W. Roberts, Control Chart Test Based on Geometric Moving Average, Technometrics, Vol. 42, No. 1, Special 40th Anniversary Issue (Feb., 2000), pp. 97-101.

[16] N. Ye, C. Borror and Y. Zhang, EWMA techniques for computer intrusion detection through anomalous changes in event intensity, Quality and Reliability Engineering International Journal Wily, vol. 18. pages 443-451, 2002.

[17] J. M. Lucas and M. S. Saccucci. Ewma Control Schemes: Properties and Enhancements. Technometrics, 32, Feb 1990. 